\title{
Epithelial Calreticulin Up-Regulation Promotes Profibrotic Responses and Tubulointerstitial Fibrosis Development
}

\author{
Niki Prakoura, ${ }^{*}$ Panagiotis K. Politis, ${ }^{*}$ Yoshito Ihara, ${ }^{\dagger}$ Marek Michalak, ${ }^{\ddagger}$ and Aristidis S. Charonis*
}

From the Section of Histology, * Biomedical Research Foundation of the Academy of Athens, Athens, Greece; the Department of Biochemistry, ${ }^{\dagger}$ Wakayama Medical University, Wakayama, Japan; and the Department of Biochemistry, ${ }^{\ddagger}$ University of Alberta, Edmonton, Alberta, Canada

Accepted for publication

July 22, 2013.

Address correspondence to Aristidis S. Charonis, M.D., Ph.D., Section of Histology, Biomedical Research Foundation of the Academy of Athens, 4 Soranou Efesiou, 11527

Athens, Greece. E-mail: acharonis@bioacademy.gr.

\begin{abstract}
Renal fibrosis is the common anatomical feature underlying the progression of chronic kidney disease, a leading cause of morbidity and mortality worldwide. In a previous study, we demonstrated that during development of renal fibrosis in a rat model of unilateral ureteric obstruction, calreticulin (CRT) is up-regulated in tubular epithelial cells (TECs). In the present study, we used in vitro and in vivo approaches to examine the role of CRT in TECs and its contribution to the progression of fibrosis. In cultured renal TECS, CRT overexpression induced acquisition of an altered, profibrotic cellular phenotype. Consistently, the opposite effects were observed for CRT knockdown. Subsequently, we confirmed that critical changes observed in vitro were also apparent in tubular cells in vivo in the animal model of unilateral ureteric obstruction. In agreement with these results, we demonstrate that substantial $(50 \%)$ reduction in the expression of CRT reduced the development of tubulointerstitial fibrosis at a comparable level through regulation of inflammation, transcriptional activation, transforming growth factor $\beta 1$-associated effects, and apoptosis. In summary, our findings establish that CRT is critically involved in the molecular mechanisms that drive renal fibrosis progression and indicate that inhibition of CRT expression might be a therapeutic target for reduction of fibrosis and chronic kidney disease development. (Am J Pathol 2013, 183: 1474-1487; http:// dx.doi.org/10.1016/j.ajpath.2013.07.014)
\end{abstract}

Chronic kidney disease (CKD), leading eventually to renal failure and the need for replacement therapy, is frequently observed in all adult populations, affecting $>10 \%$ of individuals. $^{1,2}$ Although CKD is the end result of several renal and systemic diseases, its common anatomical feature is progressive development of fibrosis. This is a complex process involving many cell types, of which the phenotype becomes modified, and cascades of overlapping biochemical pathways. $^{3-6}$ Currently, several aspects of these events are poorly understood.

Previously we have performed proteomic analysis of renal parenchyma of the well-established rodent model of unilateral ureteric obstruction (UUO). ${ }^{7,8}$ Our initial studies indicated that calreticulin (CRT) is among the proteins that are consistently up-regulated from the earliest stages of the process of renal fibrosis, even before the extracellular matrix (ECM) begins to accumulate. ${ }^{9}$ Up-regulation was confirmed at the protein and mRNA levels and was localized exclusively in tubular epithelial cells (TECs).
CRT is a multifunctional endoplasmic reticulum (ER) protein that acts as a molecular chaperone and $\mathrm{Ca}^{2+}$-binding molecule. However, it has also been found in other cellular compartments such as the cell surface and in the extracellular environment. Altered expression or localization of CRT alters cellular functions including the $\mathrm{Ca}^{2+}$ storage capacity of ER, cell adhesiveness, proliferation and apoptosis, gene expression, and immune system function. ${ }^{10,11}$ Furthermore, it is essential for cardiac development and mouse embryogenesis. ${ }^{12}$ Recent studies have implicated CRT in wound healing and chronic kidney transplant rejection. ${ }^{11,13}$ However, our initial observation was the first to involve CRT in the fibrotic process. ${ }^{9}$

Supported by the European Union (European Social Fund-ESF) and Greek national funds through the Operational Program Education and Lifelong Learning of the National Strategic Reference Framework-Research Funding Program Heracleitus II; ARISTEIA grant No. 2681 (Renal Fibrosis) from the General Secretariat of Research and Technology of the Ministry of Education (A.C.); and by funds from the Biomedical Research Foundation of the Academy of Athens. 
In the present study, using an in vitro approach, we investigated alterations in the cellular phenotype when CRT expression was increased in cultured renal TECs. We demonstrated that these cells acquired a more mesenchymal character reminiscent of initial steps of epithelialmesenchymal transition (EMT), exhibited excessive and selective ECM production, and showed increased ER stress and apoptosis. Consistently, significant reduction of CRT expression by shRNA-mediated knockdown suppressed both profibrotic and proinflammtory markers. Subsequently, we examined and confirmed that some of the observed changes in vitro were also apparent in tubular cells in vivo in the animal model of UUO. In addition, we evaluated the effect of drastic reduction in expression of CRT in heterozygous transgenic mice ${ }^{14}$ on development of fibrosis in the UUO model and observed that reduction of CRT conferred substantial protection and attenuated the process of tubulointerstitial fibrosis.

\section{Materials and Methods}

\section{Cell Culture}

Human proximal TECs (HK-2) were purchased from ATCC (Manassas, VA). The cells were grown 1:1 in [Dulbecco's modified Eagle's medium $4.5 \mathrm{~g} / \mathrm{L}$ glucose and F-12 supplemented with $10 \%$ fetal bovine serum (FBS), $100 \mu \mathrm{g} / \mathrm{mL}$ penicillin-streptomycin, and $2 \mathrm{mmol} / \mathrm{L} \mathrm{L}$-glutamine] and were subcultured according to the manufacturer's instructions.

CRT-overexpressing HK-2 cell lines were generated via stable transfection of pcDNA3.1/hygro plasmid carrying mouse CRT cDNA (kindly provided by Dr. Yoshito Ihara, Wakayama Medical School, Japan). Control cells were transfected with the empty vector. In brief, HK-2 cells were transfected in six-well plates with pcDNA3.1-hygro-mouse CRT or empty vector using lipofectamine 2000 transfection reagent (Invitrogen Corp., Carlsbad, CA). After 2 days, the cells were transferred to $10-\mathrm{mm}$ culture dishes and grown in normal medium plus $200 \mu \mathrm{g} / \mathrm{mL}$ hygromycin B (Merck \& Co., Inc., Whitehouse Station, NJ), which was renewed every 2 or 3 days. After 10 to 12 days, stable clones were removed and individually expanded. The expression level of CRT in each clone was examined via Western blotting. The selected clones were then maintained in normal growth medium supplemented with $100 \mu \mathrm{g} / \mathrm{mL}$ hygromycin.

CRT knockdown HK-2 cell lines were generated with stable transfection of pSuper.retro.puro vector carrying an shRNA sequence specific for human CRT. Control cells were transfected with the empty vector. In brief, shRNA sense and antisense sequences were annealed and cloned into pSuper.retro.puro vector in BglII-XhoI restriction sites. HK-2 cells were transfected in six-well plates with pSuper.retro.puro-CRT shRNA or empty vector, as above. Stable clones were selected in $2 \mu \mathrm{g} / \mathrm{mL}$ puromycin (AppliChem GmbH, Darmstadt, Germany). The expression level of CRT in each clone was examined via Western blotting.
The selected clones were then maintained in normal growth medium supplemented with $1 \mu \mathrm{g} / \mathrm{mL}$ puromycin. The sequences for human CRT-specific shRNA were as follows: 5'-GATCCCCGGAGCAGTTTCTGGACGGATTCAAGAGATCCGTCCAGAAACTGCTCCTTTTTC-3' and 5'-TCGAGAAAAAGGAGCAGTTTCTGGACGGATCTCTTGAATCCGTCCAGAAACTGCTCCGGG-3'.

For transforming growth factor beta1 (TGF- $\beta 1$ ) treatment of the CRT knockdown cells, the cells were incubated with or without $5 \mathrm{ng} / \mathrm{mL}$ TGF- $\beta 1$ (Sigma-Aldrich, St. Louis, MO) in serum-deprived medium for 48 hours, after which they were collected for Western blotting or quantitative RTPCR (RT-qPCR) analysis.

\section{Animals}

Eight- to 12-week-old male Wistar rats were supplied from the colony of our Center for Experimental Surgery. For the UUO model, rats underwent ligation of the right ureter, and sham-operated rats were used as controls, as described previously. ${ }^{9}$

CRT heterozygous $\left(\mathrm{CRT}^{+/-}\right) \mathrm{C} 57 \mathrm{BL} / 6$ mice, expressing only one functional CRT allele, and their wild-type (WT) littermates were provided by Dr. Marek Michalak (University of Alberta, Canada) and have been previously described. ${ }^{14}$ For the UUO model, 12-week-old $C R T^{+/-}$and WT male mice underwent ligation of the right ureter, and the contralateral kidneys served as controls. All aspects of animal experimentation were performed in adherence to the $\mathrm{NIH}$ and the European Union Guide for the Care and Use of Laboratory Animals and were approved by the Institutional Review Board of the Biomedical Research Foundation of the Academy of Athens.

\section{Western Blot Analysis}

Proteins from cell cultures and tissue samples were extracted in radioimmunoprecipitation assay buffer [50 $\mathrm{mmol} / \mathrm{L}$ Tris $(\mathrm{pH} \mathrm{7.4)}, 1 \% \mathrm{NP}-40,0.25 \%$ deoxycholate (DOC), $150 \mathrm{mmol} / \mathrm{L} \mathrm{NaCl}, 1 \mathrm{mmol} / \mathrm{L} \mathrm{Na}_{2}$ EDTA, $1 \mathrm{mmol} / \mathrm{L}$ phenylmethylsulfonyl fluoride, and cocktail protease inhibitors] and measured for protein concentration using the Bradford method, and equal protein amounts were loaded in an SDS-PAGE apparatus, followed by Western blotting. The primary antibodies used were CRT (catalog No. 06661; Upstate Biotechnology, Inc., Lake Placid, NY), Ecadherin (BD 610181; BD Biosciences, San Jose, CA), vinculin (Sigma V9131; Sigma), vimentin (catalog No. MS-129; Thermo Scientific, Inc., Waltham, MA), GRP78 (AP06149PU-N; Acris Antibodies GmbH, Herford, Germany), fibronectin (sc-8422; Santa Cruz Biotechnology, Inc., Santa Cruz, CA), COL1A1 (sc-25974; Santa Cruz Biotechnology), phospho-Smad3 (ab52903; Abcam, Cambridge, England), Smad3 (catalog No. 3102; Cell Signaling Technology, Inc., Danvers, MA), $\beta$-actin (catalog No. A5316; Sigma), and $\beta$-tubulin (ab6046; Abcam). 
For Col1A1 immunoblotting in conditioned medium, cells were cultured for 24 hours in normal growth medium and then in serum-deprived medium for 48 hours. Conditioned medium was then collected with protease inhibitors, centrifuged to pellet cells, and concentrated three times in a Concentrator 5301 (Eppendorf AG, Hamburg, Germany). Equal volumes from different samples were loaded in a normal SDS-PAGE apparatus, followed by Western blotting. For evaluation of CollA1 levels separately in the cellular and ECM compartments, DOC separation of the fractions was performed according to the method of Van Duyn Graham et al. ${ }^{15}$ In brief, cells were seeded in six-well plates, cultured for 24 to 48 hours in normal growth medium, and then in serum-deprived medium for another 48 hours. The cells and ECM were washed with PBS and harvested by scraping in 4\% DOC solution [4\% DOC in 20 $\mathrm{mmol} / \mathrm{L}$ Tris $\mathrm{HCl}(\mathrm{pH} 8.8)$, with $1 \times$ protease inhibitors]. Lysates were homogenized with a 27-1/2-gauge needle and pelleted at $13,000 \mathrm{rpm}$ for 30 minutes at $4^{\circ} \mathrm{C}$. The supernatant was kept as the DOC-soluble cellular fraction, and the pellet containing the DOC-insoluble ECM fraction was washed with $4 \%$ DOC solution and resolubilized in $1 \%$ SDS, $25 \mathrm{mmol} / \mathrm{L}$ Tris $\mathrm{HCl}$ ( $\mathrm{pH} 8.0$ ), plus protease inhibitors. The total DOC insoluble fraction and one-tenth of the DOCsoluble fraction were loaded in a normal SDS-PAGE apparatus, followed by Western blotting. Each experiment was performed in duplicate or triplicate and was repeated three times.

\section{RT-qPCR}

Total RNA was prepared from cultured cells and mouse kidney tissue using TRI reagent (Molecular Research Center, Cincinnati, OH) according to manufacturer's instructions. For the cell preparations, cells were seeded in six-well plates in normal growth medium and were cultured for 48 to 72 hours until they were near confluence. The quality of the RNA was verified on agarose gels, and the quantity was measured using a Spectronic photometer (Thermo Fisher Scientific). Total RNA, $1 \mu \mathrm{g}$, was reverse transcribed using ImProm-II Reverse Transcriptase (Promega Corp., Madison, WI). We performed RT-qPCR using ABI Prism 7000 software version 1.1 (Applied Biosystems, Inc., Foster City, CA), with Platinum TaqDNA polymerase (Invitrogen), three-step standard cycling conditions, and sequence-specific primers. We performed a melting curve analysis to verify that a single product was amplified. For quantitative analysis, experimental genes were normalized to $18 \mathrm{~S}$ rRNA or GAPDH gene expression using the $\Delta \Delta \mathrm{CT}$ method. The experiments were performed in triplicate and were repeated three times.

\section{Proliferation Assay}

Cell proliferation was studied using a BrdU-incorporation immunolabeling assay. Cells were seeded on coverslips and cultured for 24 to 48 hours. After 2 hours of incubation with BrdU solution, the cells were fixed in $4 \%$ paraformaldehyde, permeabilized with $2 \mathrm{~N} \mathrm{HCl}$, blocked with $5 \% \mathrm{FBS}$, and incubated with a primary anti-BrdU antibody (ab6326; Abcam). A fluorescent secondary antibody (anti-rat Alexa Fluor; Invitrogen) was used for detection of the BrdUpositive signal. The cells were counterstained with DAPI for nuclei visualization and mounted on slides in Mowiol 4-88 reagent (Calbiochem, EMD Millipore, Billerica, MA). Random non-overlapping images were obtained at $\times 200$ magnification with the use of a fluorescent microscope (DM RA2; Leica Microsystems GmbH, Wetzlar, Germany) followed by manual counting of BrdU-positive signals in 500 cells per sample. Each experiment was performed in duplicate or triplicate and was repeated three times. Data were expressed as percentage of BrdU-DAPI ratio.

\section{Apoptosis Assay}

Apoptosis was assessed using a fluorescein TUNEL kit (Roche Applied Science, Penzberg, Upper Bavaria, Germany), according to manufacturer's instructions. To sensitize cells with $\mathrm{H}_{2} \mathrm{O}_{2}$ before apoptosis assessment, cells were incubated with 50 $\mu \mathrm{mol} / \mathrm{L} \mathrm{H}_{2} \mathrm{O}_{2}$ in growth medium for 2 hours. In brief, paraformaldehyde-fixed cells on coverslips or rehydrated formalin-fixed, paraffin-embedded (FFPE) mouse kidney sections were permeabilized with $0.1 \%$ Triton $\mathrm{X}-100$ in 10 $\mathrm{mmol} / \mathrm{L}$ sodium citrate $(\mathrm{pH} 6.0)$, followed by incubation with TUNEL reaction mixture for 1 hour at $37^{\circ} \mathrm{C}$. Samples were counterstained with DAPI and mounted in Mowiol 4-88 reagent (Calbiochem). Random non-overlapping images were obtained at $\times 200$ magnification using a fluorescent microscope (DM RA2; Leica) followed by manual counting of TUNEL-positive signals in 500 cells per sample for the cell experiments or in 8 to 10 high-power fields per sample for the tissue experiments. Each experiment was performed in duplicate or triplicate and was repeated two or three times. Cell data were expressed as percentage of TUNEL-DAPI counts, and tissue data were expressed as tubular TUNEL counts per high-power field.

Cleaved-caspase 3 staining was also indicative of apoptosis in cultured cells (see Immunofluorescence).

\section{Motility Assay}

Cell motility was assessed using a wound-healing closure assay. The cells were cultured in 48 -well plates in duplicate until they reached confluence. Then the growth medium was replaced with serum-deprived medium at 24 hours before wound induction. Three hours before wound induction, mitomycin $\mathrm{C}$ was added to the cells at a final concentration of $10 \mu \mathrm{g} / \mathrm{mL}$ in serum-deprived medium, to stop cell proliferation. To create the wound, a plastic pipette tip was gently drawn across the center of the well to produce a clean wound approximately $1 \mathrm{~mm}$ wide. Images of the cells were obtained at $\times 50$ magnification using an Axiovert 200 microscope (Carl Zeiss GmbH, Jena, Germany) at 0, 24, 48, 
and 72 hours after wound induction. To quantify cell migration, the wounded area was measured using ImageJ version 1.43 (NIH, Bethesda, MD). The experiment was repeated three times. Data were expressed as the percentage of the remaining wounded area at each time point.

\section{Immunofluorescence}

Cells were seeded on coverslips in 24-well plates in duplicate and cultured for 24 to 48 hours. To induce oxidative stress before cleaved caspase-3 staining, cells were incubated with $50 \mu \mathrm{mol} / \mathrm{L} \mathrm{H}_{2} \mathrm{O}_{2}$ in growth medium for 2 hours. The coverslips were removed from the wells, and the cells were fixed in $4 \%$ paraformaldehyde, blocked with a $5 \%$ FBS solution, incubated with primary antibodies to collagen IV (C1926; Sigma) or cleaved caspase-3 (catalog No. 9661; Cell Signaling Technology), developed using Alexa Fluor fluorescent secondary antibodies (Invitrogen), counterstained with DAPI, and mounted in Mowiol 4-88 reagent (Calbiochem). Random non-overlapping images were obtained at $\times 200$ magnification using a fluorescent microscope (Leica DM RA2). For quantification of cleaved caspase-3 staining, 500 cells per sample were manually counted. The experiments were repeated three times.

For dual immunolabeling, FFPE rat kidney sections were rehydrated, treated for antigen retrieval with $10 \mathrm{mmol} / \mathrm{L}$ sodium citrate $(\mathrm{pH} 6.0)$ at $100^{\circ} \mathrm{C}$ for 10 minutes, blocked with a $10 \%$ FBS solution, incubated with primary antibodies to GRP78 (AP06149PU-N; Acris Antibodies)/CRT (catalog No. 06-661; Upstate Biotechnology) developed using Alexa Fluor fluorescent secondary antibodies (Invitrogen), counterstained with DAPI, and mounted in Mowiol 4-88 reagent (Calbiochem). Double immunofluorescence images were obtained using a Leica inverted confocal microscope.

For $\alpha$-SMA immunostaining, FFPE mouse kidney sections were rehydrated, treated for antigen retrieval with $10 \mathrm{mmol} / \mathrm{L}$ sodium citrate $(\mathrm{pH} 6.0)$ at $80^{\circ} \mathrm{C}$ for 20 minutes, blocked with a $10 \%$ FBS solution, incubated with anti$\alpha$-SMA primary antibody (Abcam), developed using Alexa Fluor fluorescent secondary antibody (Invitrogen), counterstained with DAPI, and mounted in Mowiol 4-88 reagent (Calbiochem). Non-overlapping images of the entire cortex were obtained using a Leica inverted confocal microscope at $\times 400$ magnification. The $\alpha$-SMA-positive area (expressed as percentage of total area excluding large vessels) was quantified in all of the images using publicly available image processing software (ImageJ Fiji, a distribution of ImageJ; http://fiji.sc/Fiji).

\section{Histology}

FFPE kidney sections stained with Sirius Red were used for histologic analysis. Slides were examined, and nonoverlapping images of the entire cortex were obtained using a Leica DM LS2 microscope and a Leica DFC 500 camera at $\times 400$ magnification. The tubulointerstitial area stained (expressed as percentage of total area excluding glomeruli and large vessels) was quantified in all of the images using ImageJ Fiji.

\section{Statistical Analysis}

Results are presented as means $\pm \mathrm{SD}$. To analyze the difference between two data sets, the two-tailed Student's $t$-test was used, and for multiple group comparisons, analysis of variance with post hoc analysis. $P<0.05$ was considered significant.

\section{Results}

\section{Altered CRT Expression in TECs Influences Their Motility, Stress, Apoptosis, and Proliferation}

To understand how CRT up-regulation in the tubular cells of the kidney affects the progression of fibrosis, we analyzed the phenotype of both CRT-overexpressing and CRT knockdown TECs. For overexpression, we used an established proximal TEC line (HK-2) and created HK-2 cell lines that stably overexpressed mouse CRT cDNA, using one cell line with low and one with high CRT overexpression, as well as one control line transfected with the control vector (Figure 1A). Compensatory down-regulation of the human CRT transcript was observed for the cell line with the greater total CRT expression, possibly explaining why higher overexpression folds were not achieved (Supplemental Figure S1). For knockdown, we created HK-2 cell lines that stably expressed an shRNA molecule specifically targeting human CRT and used two cell lines
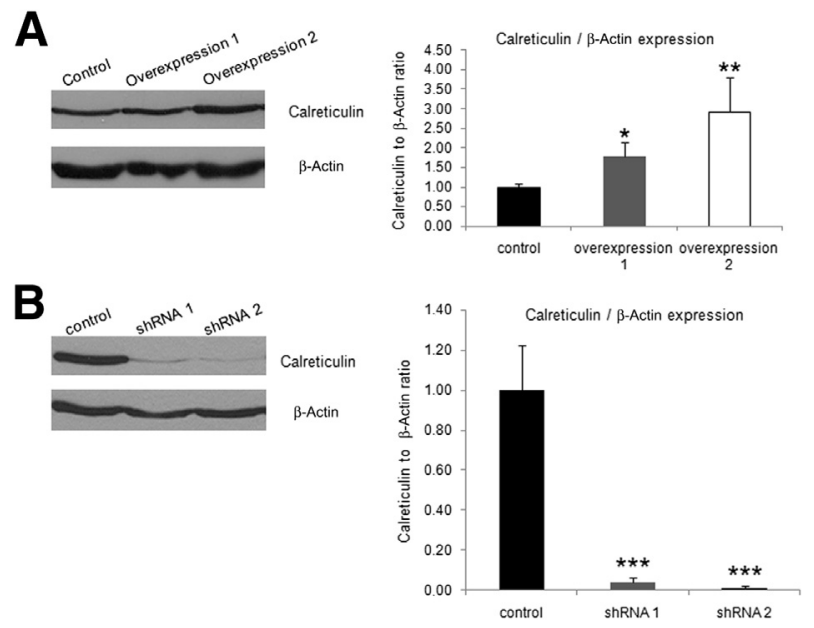

Figure 1 Calreticulin (CRT) overexpression and knockdown in tubular epithelial cells (TECS). Western blot normalized to $\beta$-actin expression and corresponding quantification of CRT-overexpressing cells (A) and CRT knockdown TECs (B). Control, cell line transfected with control vector; overexpression 1 or overexpression 2, cell line with overexpression. Quantification corresponds to means \pm SD of three independent experiments. ${ }^{*} P<0.05,{ }^{* *} P<0.01$, and ${ }^{* * *} P<0.001$ versus control. 


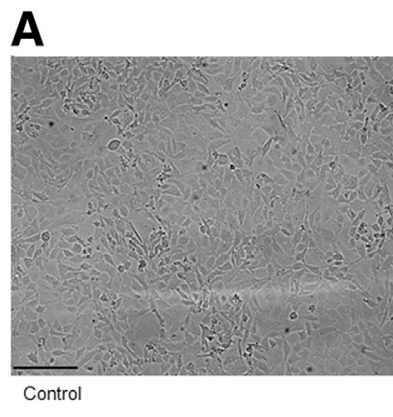

B

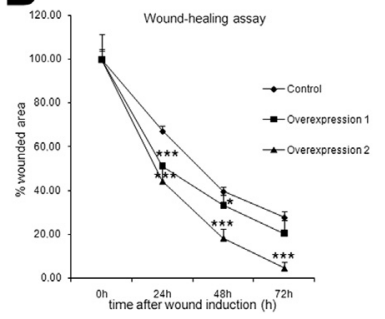

C

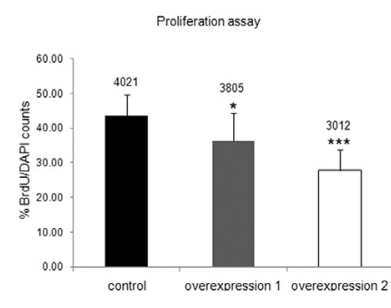

Figure 2 Calreticulin (CRT) overexpression alters the structure, motility, and proliferative capacity of tubular epithelial cells (TECs). A: Representative images of control and CRT-overexpressing TECs in culture show loss of epithelial adhesion and acquisition of a more elongated, mesenchymal-like structure due to CRT overexpression. B: Wound-healing assay in the presence of mitomycin $C$ to inhibit proliferation shows increased motility and wound closure of CRT-overexpressing cells up to 72 hours after wound induction. C: BrdU-incorporation assay shows decreased proliferation of CRT overexpressors. Results are expressed as percentage of BrdU-DAPI ratio for each cell line. The number of DAPI-positive cells counted for each cell line is indicated above the respective graph bars. Data represent means $\pm \mathrm{SD}$ of three independent experiments. ${ }^{*} P<0.05,{ }^{*} P<$ 0.01 , and ${ }^{* *} P<0.001$ versus control. Scale bars: $250 \mu \mathrm{m}$ (A).

with significant knockdown of CRT expression and one control line transfected with the empty vector (Figure 1B).

It was observed that, compared with control cells, CRToverexpressing TECs were more elongated and mesenchymallike and adhered more loosely to each other (Figure 2A). Next we examined motility using a wound-healing closure assay. Compared with control cells, CRT-overexpressing cell lines displayed significantly increased motility up to 72 hours after wound induction, proportional to the level of CRT expression (Figure 2B). This phenomenon was not due to an increase in proliferation rate because mitomycin $\mathrm{C}$ was added to the assay to differentiate proliferation from migration. Moreover, in BrdU-incorporation assays, the overexpressing cell lines showed reduced proliferation (Figure 2C). The smaller total number of DAPI nuclei counted in CRT-overexpressing cells compared with control cells (Figure 2C) suggests that the overexpressing cells proliferate more slowly.

Next we studied the intracellular stress state by examining the expression levels of GRP78, a major ER stress sensor. ${ }^{16}$ CRT-overexpressing cells displayed significantly increased levels of GRP78, showing that CRT up-regulation induces ER stress response mechanisms (Figure 3A). Inasmuch as ER stress progression can lead to apoptosis and cell death, ${ }^{17}$ we assessed the levels of cellular apoptosis using the
TUNEL assay, which detects DNA fragmentation. Compared with control cells, CRT-overexpressing cells showed a twofold increase in apoptosis under normal culture conditions. After treatment with $\mathrm{H}_{2} \mathrm{O}_{2}$, apoptosis of the overexpressing cells was dramatically increased, in particular for the clone with greater CRT expression (Figure 3B). The total number of DAPI-positive cells counted for the TUNEL assay was reduced in the overexpressing cells compared with control cells, in particular after treatment with $\mathrm{H}_{2} \mathrm{O}_{2}$ (Figure $3 \mathrm{~B}$ ), which suggests that CRT overexpressors died massively in response to $\mathrm{H}_{2} \mathrm{O}_{2}$. In addition, cleaved caspase-3 expression, which detects activation of the execution phase of apoptosis, assessed via immunostaining, was increased in the overexpressing cells in the absence or presence of $\mathrm{H}_{2} \mathrm{O}_{2}$ in the growth medium (Figure 3C). The difference between cleaved caspase3 -positive and TUNEL-positive cells after $\mathrm{H}_{2} \mathrm{O}_{2}$ treatment may indicate that there are caspase-3-independent mechanisms that induce DNA fragmentation in the CRT overexpressors under conditions of oxidative stress. Moreover, the higher level of unstimulated apoptosis due to CRT
A
B

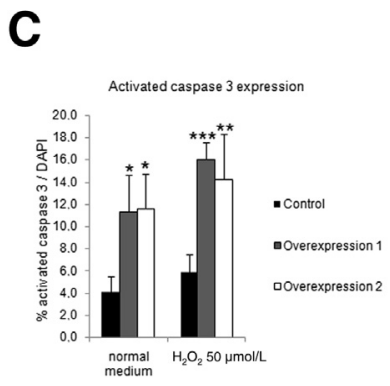

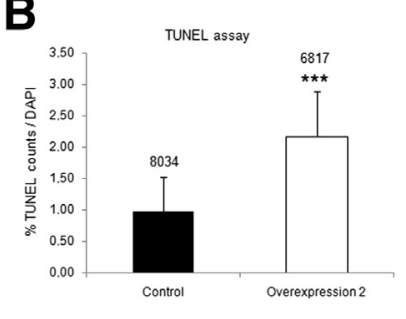
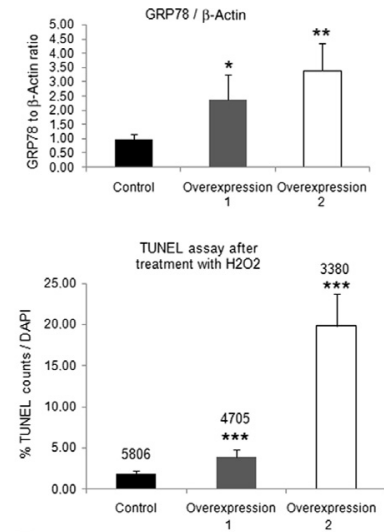

D

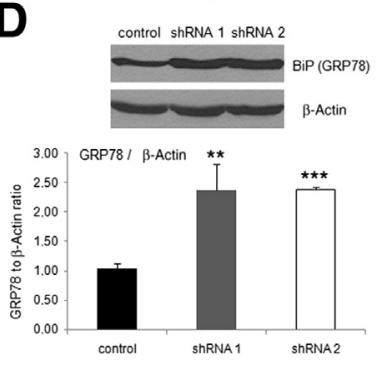

Figure 3 Calreticulin (CRT) overexpression in tubular epithelial cells (TECs) induces endoplasmic reticulum (ER) stress and cellular apoptosis mechanisms. A: Representative blot shows increased GRP78 expression, an endoplasmic reticulum stress sensor, in CRT-overexpressing cells. Quantification corresponds to means \pm SD of three independent experiments. TUNEL-positive nuclei-DAPI ratio (B) and cleaved caspase-3-DAPI ratio (C) in control and CRT-overexpressing cells under normal growth conditions or after treatment with $50 \mu \mathrm{mol} / \mathrm{L} \mathrm{H}_{2} \mathrm{O}_{2}$ for 2 hours. The number of DAPIpositive cells counted for each cell line is indicated above the respective graph bars. Data represent means \pm SD of three independent experiments. D: Representative blot shows increased GRP78 expression in CRT knockdown cells. Quantification corresponds to means \pm SD of three independent experiments. ${ }^{\star} P<0.05,{ }^{* *} P<0.01$, and ${ }^{* * *} P<0.001$ versus control. 

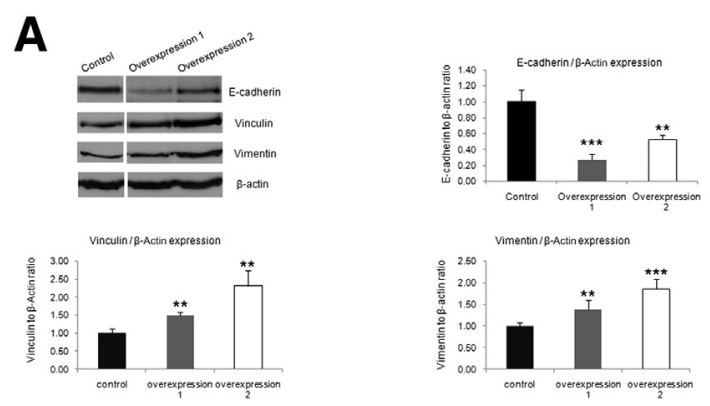

B
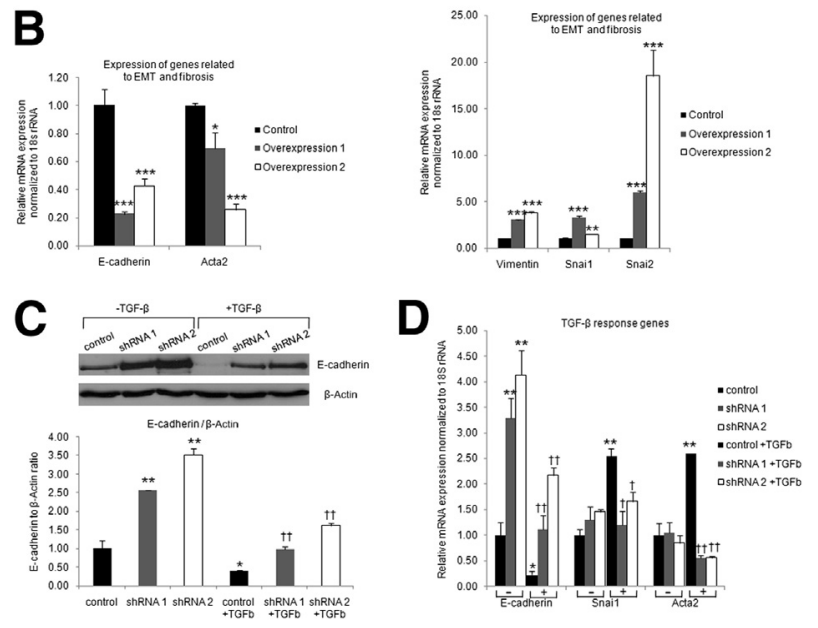

Figure 4 Altered expression of epithelial and mesenchymal markers caused by calreticulin (CRT) overexpression or knockdown in tubular epithelial cells (TECS). A: Representative blots show substantially reduced E-cadherin and increased vimentin and vinculin expression in CRT-overexpressing cells. The lanes were run on the same gel but were noncontinguous. Quantification corresponds to means \pm SD of three independent experiments. B: Relative mRNA amount (RT-qPCR) of several EMT markers (E-cadherin, Vimentin, Acta2, Snai1, Snai2) in control and CRT-overexpressing cells. C: Representative blot of E-cadherin expression in TECs underexpressing CRT with or without TGF- $\beta 1$ treatment. E-cadherin is remarkably increased in knockdown cells, and its suppression by TGF- $\beta 1$ is compromised. D: Relative mRNA amount of TGF- $\beta 1$ response genes in CRT knockdown cells. CRT suppression impairs TGF- $\beta 1$ signaling in TECs. Data represent means \pm SD of three independent experiments. ${ }^{*} P<0.05,{ }^{* *} P<0.01$, and ${ }^{* * *} P<0.001$ versus control; ${ }^{\dagger} P<0.05$, ${ }^{\dagger} P<0.01$ versus control + TGF- $\beta$.

overexpression observed in both TUNEL and caspase-3 assays may be attributed to the increased expression of activated caspase- 3 in the overexpressing cells or may be induced through ER stress mechanisms triggered by CRT overexpression.

In the CRT knockdown cells, a significant increase in GRP78 levels was also observed (Figure 3D), probably to compensate for the considerable CRT suppression. However, the function of GRP78 and the purpose of ER stress induction likely differ between CRT knockdown and overexpressing cells. Consistently, CRT knockdown cells did not display any important change in apoptosis or viability rates in TUNEL, caspase-3, or BrdU assays, features that could be influenced by ER stress (data not shown). It seems that reduction of CRT levels is not so crucial for proliferation and apoptosis of TECs as is its overexpression. Moreover, the unaltered activated caspase-3 expression may account for the failure to observe protection from apoptosis of the CRT knockdown cells (data not shown).

\section{CRT Overexpression or Knockdown in TECs Alters Expression of Epithelial and Mesenchymal Markers}

Because progression of fibrosis has been associated with changes in the epithelial cell phenotype, ${ }^{5,6}$ we examined the expression levels of epithelial and mesenchymal protein markers in both CRT-overexpressing and knockdown TECs. The epithelial junctional protein E-cadherin was substantially decreased in the overexpressing cells. In parallel, mesenchymal proteins such as vinculin and vimentin were increased (Figure 4A). These results showed that CRT upregulation causes a decline of the epithelial cell phenotype in favor of a more mesenchymal character.

To confirm previous findings, we further characterized several markers at the gene expression level. E-cadherin and vimentin expression followed the same pattern as did protein expression (Figure 4B). Snail (snail) and Snai2 (slug), two transcriptional repressors of E-cadherin and strong inducers of EMT, ${ }^{18-20}$ were significantly up-regulated in CRToverexpressing cells. However, expression levels of Acta2 (the gene name for $\alpha$-SMA), the most commonly used marker for myofibroblasts, were decreased in the CRT overexpressors, indicating that despite their phenotypic change, CRT-overexpressing TECs are not promoted to reach EMT.

CRT knockdown led to an important increase in E-cadherin expression at both the protein (Figure 4C) and mRNA (Figure 4D) levels, showing the opposite phenotype as the overexpressing TECs. Other EMT markers such as Snail and Acta2 were not changed by CRT suppression (Figure 4D). However, induction of both Snail and Acta2 after TGF- $\beta 1$ treatment was completely abolished in the CRT knockdown TECs. Furthermore, suppression of E-cadherin induced by TGF- $\beta 1$ was partially attenuated (Figure 4D). These results show that down-regulation of CRT expression inhibits several TGF- $\beta 1$-driven responses of epithelial cells in culture, supporting a profibrotic role of CRT.

\section{Altered CRT Expression Levels Change the Secretory Profile of TECs}

As fibrosis development is highly based on ECM accumulation, we tested whether CRT up-regulation or knockdown in TECs affects the production of ECM components. Fibronectin expression was significantly increased in the CRToverexpressing cells, at both the protein and mRNA levels (Figure 5, A and D). In addition, collagen IV, an epithelial collagen type and basic component of the tubular basement membrane, was notably up-regulated in the CRT overexpressors at both the protein and mRNA expression levels (Figure 5, B and D). However, collagen I, which is known to be produced by fibroblasts, was not increased in TECs overexpressing CRT, neither at the protein (conditioned 
medium, cell, and ECM fractions) nor at the transcript level (Figure 5, C and D). That CollA1 was completely absent from the ECM fraction of TECs is in total agreement with the epithelial character of the well-differentiated cell line used and the composition of the renal tubular basement membrane. In contrast, CRT knockdown led to a dramatic decrease in Collal expression, without significantly affecting expression of Fnl or Col4al transcripts (Figure 5E). These results indicate that CRT overexpression in TECs promotes selective production of ECM components without inducing complete mesenchymal transformation of TECs; however, its suppression is able to attenuate the expression of primary mesenchymal markers such as Collal.

\section{CRT-Overexpressing Tubular Cells in Vivo Display Increased Cellular Stress}

Cellular stress originating from ER is increasingly recognized as a major mechanism contributing to the progression of renal fibrosis. ${ }^{21}$ Because we found that CRT up-regulation in cultured TECs induces ER stress, we sought to confirm whether tubular cells overexpressing CRT in vivo in the UUO model display similar characteristics. Thus, we performed double immunofluorescence staining of kidney sections from control (sham-operated) mice and at 2 or 8 days in animals who underwent ureter ligation (L2 and L8, respectively). GRP78, an indicator of ER stress, and CRT were expressed at relatively low levels in control animals (Figure 6). However, the level of both proteins gradually increased at 2 and 8 days after ureteric obstruction and displayed broad co-localization in the tubular cells of the fibrotic kidney.

CRT Heterozygous Mice Demonstrate Improved Kidney Appearance and Reduced Collagen Accumulation in the UUO Model

Because our observations indicated that CRT up-regulation in TECs induces a profibrotic cell phenotype, we used an animal model with reduced CRT expression, mice heterozygous for CRT, ${ }^{14}$ to directly investigate its role in fibrosis progression in the UUO model.

Initially, we confirmed that heterozygous mice produce approximately half the amount of CRT as their WT littermates do (Figure 7A). To analyze late stages of the disease progression, mice were sacrificed at days 8 and 17 after UUO.

At 17 days after UUO, there was a notable anatomical difference between the ligated kidneys of WT and
A

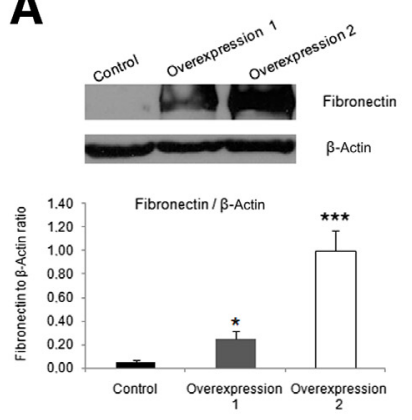

B
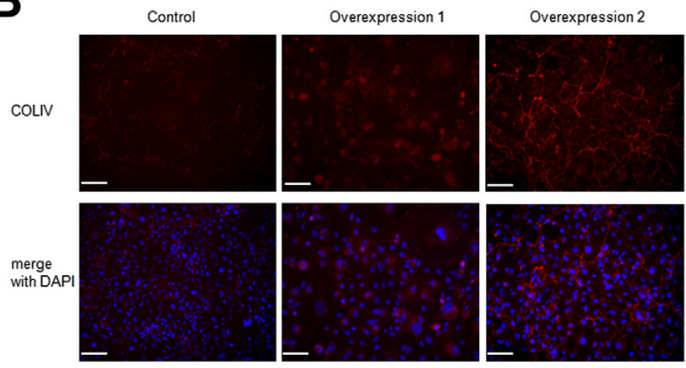

C
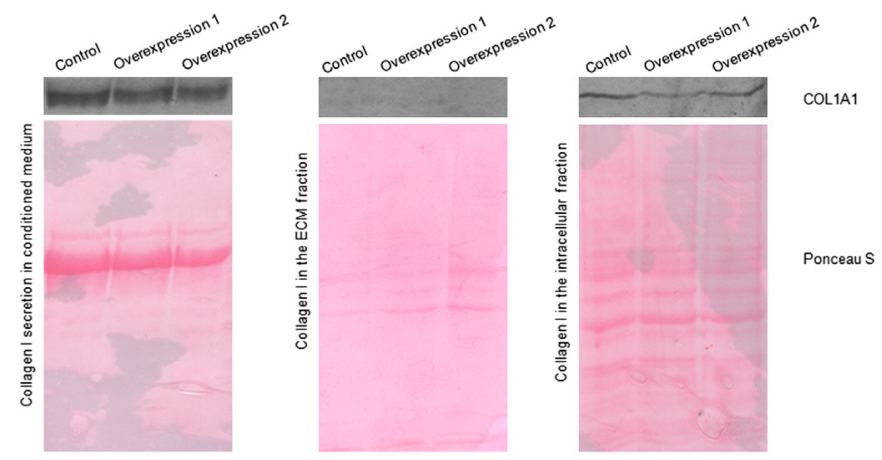

D

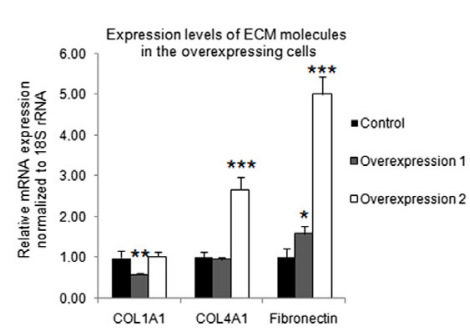

$\mathbf{E}$

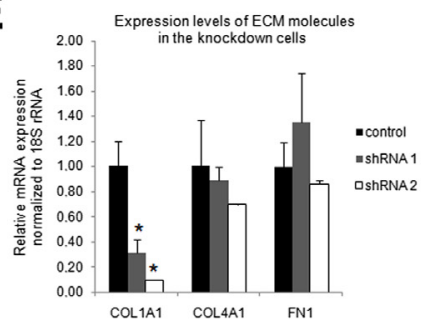

Figure 5 Calreticulin (CRT) overexpression in tubular epithelial cells (TECS) induces selective production of extracellular matrix (ECM) components. A: Representative blot shows significantly increased fibronectin expression in CRT-overexpressing cells. Quantification corresponds to means \pm SD of three independent experiments. B: Representative images of collagen IV immunostaining on control and CRToverexpressing cells show marked up-regulation in overexpressors. C: Representative blots show COL1A1 expression in conditioned medium, ECM, and cell fractions of control, and CRT-overexpressing cells. Ponceau $S$ staining was used as loading control. COL1A1, a fibroblast-specific collagen, is not increased by CRT up-regulation in TECs. D: Relative mRNA amount (RT-qPCR) of Col1a1, Col4a1, and Fn1. Transcript levels follow the same pattern as protein expression. E: Relative mRNA amount (RT-qPCR) of ECM molecules in CRT knockdown TECs shows significant suppression of Col1a1 and no change in Col4a1 and $F n 1$. Data represent means \pm SD of three independent experiments. ${ }^{*} P<0.05,{ }^{* *} P<0.01$, and $* * * P<0.001$ versus control. Scale bars: $100 \mu \mathrm{m}$ (B). 


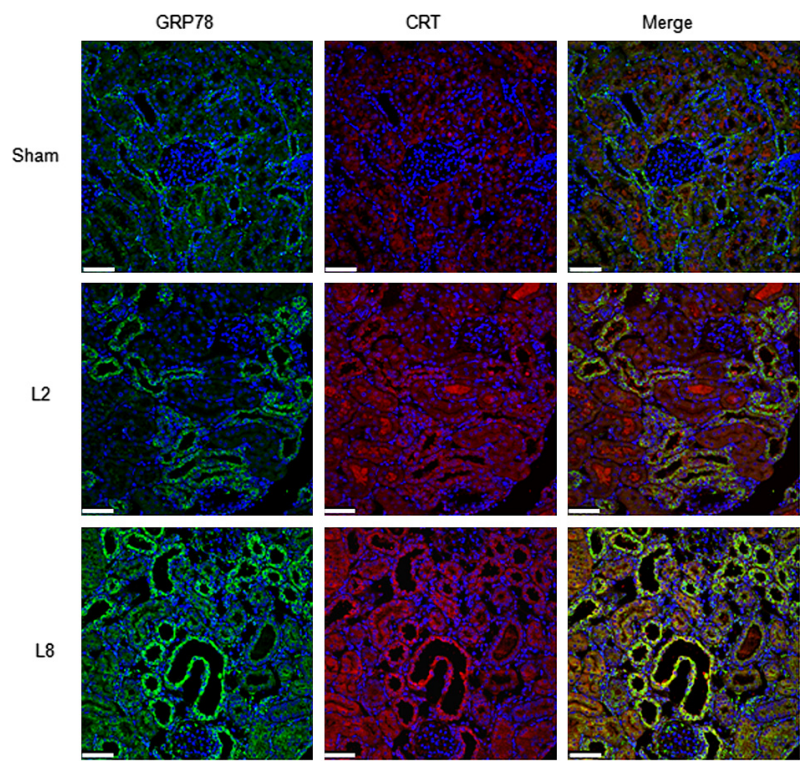

Figure 6 Increased cellular stress characterizes calreticulin (CRT)overexpressing tubular cells in vivo. Representative double immunostaining images of GRP78 (green) and CRT (red) in control (sham-operated) mice and at 2 or 8 days after ureteric ligation in study animals (L2 and L8, respectively). Both GRP78 and CRT are expressed at low levels in control animals but are gradually increased and highly co-localized in the tubular cells of the fibrotic kidney after 2 and 8 days of UUO (yellow), indicating that CRT-overexpressing tubular cells in vivo exhibit broad ER stress. Scale bars: $50 \mu \mathrm{m}$.

heterozygous mice. The ligated kidneys of WT mice displayed markedly severe dilatation and color change compared with the respective kidneys of CRT heterozygous mice (Figure 7B). The same phenomenon was observed to a lesser extent at 8 days after UUO (data not shown). This improved appearance of the obstructed kidneys in heterozygous mice was the first indication to positively correlate CRT abundance with fibrosis progression.

To further investigate whether CRT reduction affects ECM deposition during tissue fibrosis, we performed Sirius Red staining of kidney sections from WT and heterozygous mice. Quantification of the extent of Sirius Red staining showed that there was substantially reduced collagen accumulation in the kidneys of heterozygous mice, both at 8 and 17 days after UUO (Figure 7C). Collagen accumulation was decreased by $35 \%$ at 8 days and by $50 \%$ at 17 days, indicating that reduction in CRT expression protects from ECM deposition up to very late stages of fibrosis progression.

\section{CRT Heterozygous Mice Demonstrate Altered Expression of Key Molecules That Regulate Progression of Fibrosis}

We then examined the expression levels of several major profibrotic and proinflammatory molecules along with the expression levels of CRT in the mouse UUO model. RT-qPCR analysis showed that CRT was up-regulated with progression of fibrosis but was significantly reduced in heterozygous mice compared with WT mice. However, greater reduction was observed at 17 days after obstruction, indicating that CRT expression in heterozygous mice was not proportionally reduced at all time points (Figure 8A). In accordance with CRT expression levels, the profibrotic genes examined, including the ECM components Collal, Col3al, Col4al, and Fnl (fibronectin), the profibrotic growth factor $T G F-\beta 1$, and the transcription factors Snail and Snai2, were notably attenuated in heterozygous mice, but to a lesser extent at 8 days than at 17 days after UUO (Figure 8B). Moreover, examination of the expression levels of E-cadherin as a marker of epithelial integrity revealed an important reduction in WT mice at 17 days after obstruction, whereas its expression was not altered in heterozygous mice (Figure $8 \mathrm{C}$ ). Next, we determined the area of $\alpha$-SMApositive myofibroblasts and the expression levels of the proinflammatory mediators TNF- $\alpha$ and MCP1. At 17 days after obstruction, both $\alpha$-SMA and proinflammatory gene expression were significantly attenuated in heterozygous mice, comparable to the level of CRT reduction (Figure 8, D and E). In summary, these results indicate that CRT may positively regulate activation of profibrotic and proinflammatory pathways and that reduction of its levels is able to efficiently attenuate the progression of fibrosis.

\section{Renal Tubular Cells in CRT Heterozygous Mice Are Protected from Apoptosis during Fibrosis Progression}

Because apoptosis has a major role in tubular cell loss during fibrosis development, ${ }^{5,22}$ we investigated whether reduction of CRT affects tubular apoptosis in the UUO model. TUNEL staining of kidney sections showed that, compared with WT mice, CRT heterozygous mice demonstrate markedly less tubular apoptosis at both 8 and 17 days after obstruction (Figure 9). This decrease in tubular apoptosis is comparable to the expression level of CRT after UUO (Figure 8A).

\section{CRT Levels Affect Expression of Molecules Being Upstream or Downstream TGF- $\beta 1$ Signaling}

Inasmuch as CRT partial deficiency in heterozygous mice repressed expression of TGF- $\beta 1$, a key mediator of profibrotic events, we investigated whether CRT may regulate TGF- $\beta 1$ production and/or activation.

Thrombospondin 1 (TSP1) is a complex matricellular protein that regulates latent TGF- $\beta 1$ activation and has a role during tissue repair and fibrosis. ${ }^{23,24}$ We showed that TSPI was up-regulated along with development of fibrosis, yet its expression was significantly reduced in CRT heterozygous mice compared with WT littermates at both time points examined (Figure 10A). Next, we examined how CRT levels may affect TSP1 expression in the CRToverexpressing or knockdown TECs. CRT up-regulation induces a proportional increase in TSPI expression levels (Figure 10B). Experiments performed on CRT-knockdown 
TECs showed that CRT deficiency reduces TSPI expression significantly (Figure 10C). These results collectively indicate that CRT levels directly affect TSPI expression and thus may regulate TSP1-mediated TGF- $\beta 1$ activation.

Furthermore, we investigated the effect of CRT levels on expression of proinflammatory mediators because inflammatory cells represent the major source of TGF- $\beta 1$ in fibrosis. ${ }^{25}$ All three cytokines examined $(M C P 1, T N F-\alpha$, and $I L-8)$ were remarkably increased in the CRT-
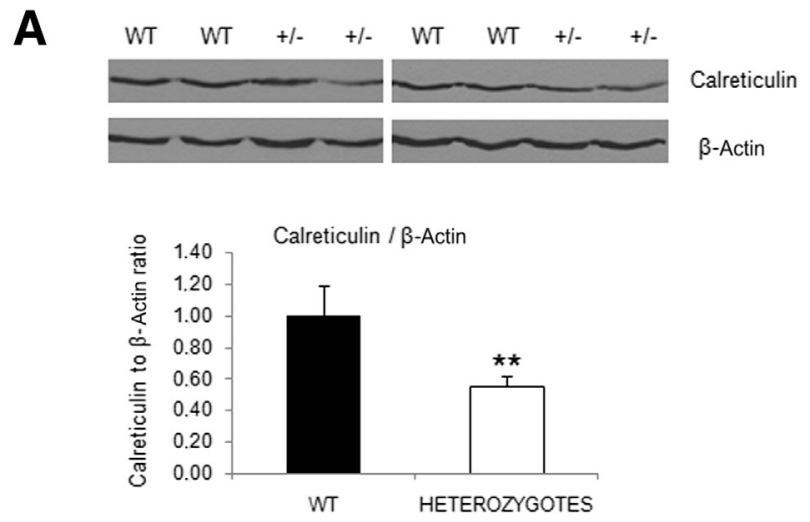

B
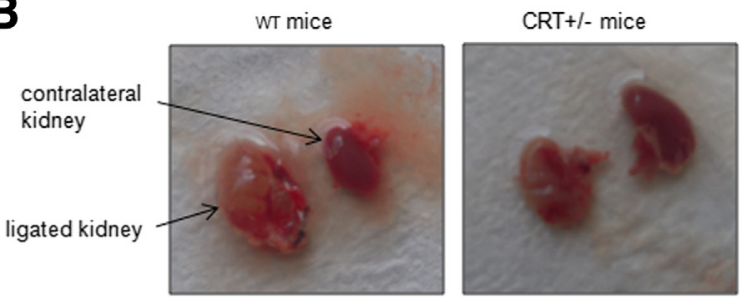

C
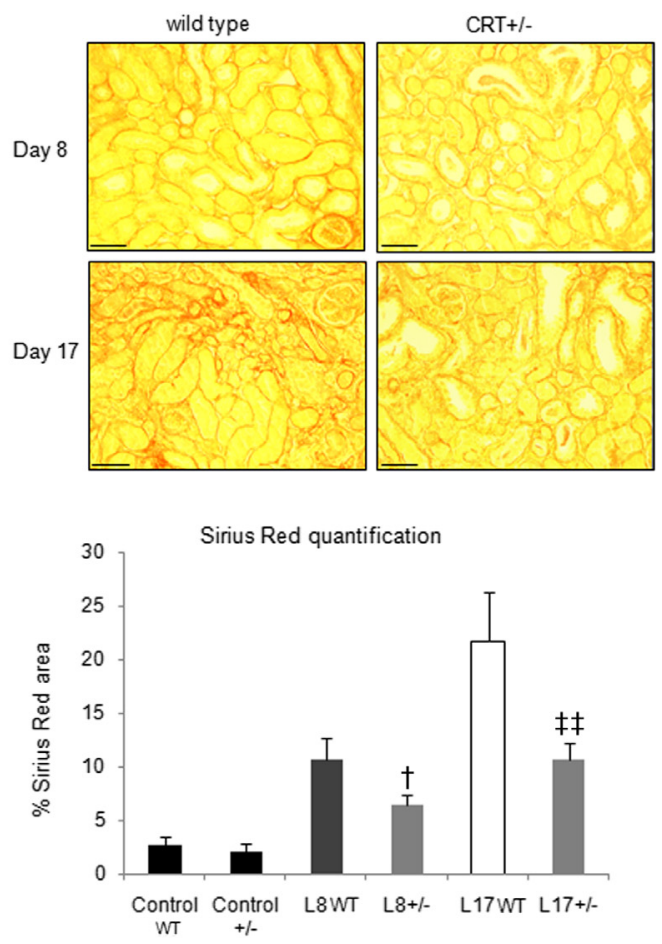

overexpressing TECs (Figure 10D). In contrast, MCPI and $T N F-\alpha$ were significantly reduced in CRT knockdown TECs (Figure 10C). This is consistent with repression of $M C P 1$ and $T N F-\alpha$ levels in CRT heterozygous mice during fibrosis progression (Figure $8 \mathrm{E}$ ) and associates CRT with regulation of inflammation and consequently TGF- $\beta 1$ production.

In agreement with these findings, we showed that phosphorylated Smad3, a central mediator of TGF- $\beta 1$ signaling, is substantially attenuated in CRT heterozygous mice at both time points of fibrosis, clearly indicating lower activation of the TGF- $\beta 1$ pathway (Figure 10E).

\section{Discussion}

Renal fibrosis is the common anatomical denominator underlying the progression of CKD of various causes. ${ }^{3}$ Only a detailed understanding of the mechanisms and macromolecules involved in fibrosis progression will enable development of targeted therapeutic interventions. In this report, we demonstrated participation of CRT during development of renal fibrosis in vitro and in vivo. Our studies in vitro with renal TECs showed that CRT upregulation was a causative factor for acquisition of an altered, primarily profibrotic cellular phenotype. Next, we confirmed that critical changes observed in vitro (cellular stress and apoptosis) were also appearing in tubular cells in vivo in the animal model of UUO. On the basis of these observations, we reasoned that reduction of CRT may exert a protective effect toward fibrosis. Indeed, we demonstrated that substantial reduction in expression of CRT in heterozygous transgenic mice impressively attenuated the progression of tubulointerstitial fibrosis.

How does CRT up-regulation in tubular cells affect progression of renal fibrosis? Overexpression of CRT in cultured renal TECs caused the decrease of differentiated epithelial cell markers (eg, E-cadherin) and acquisition of a more mesenchymal phenotype, most likely through upregulation of the key EMT regulators Snail and Snai2

Figure 7 Decreased calreticulin (CRT) expression in heterozygous mice ameliorates unilateral ureteric obstruction (UU0)-induced tubulointerstitial fibrosis. A: Representative blot of CRT expression in heterozygous mice $(+/-)$ and their WT littermates shows that the heterozygous mice produce approximately half the amount of CRT relative to their WT littermates. Quantification corresponds to means \pm SD of three independent experiments. B: Representative images of the ligated and contralateral kidneys from WT and $C R T^{+/-}$mice at 17 days after UUO shows that $C R T^{+/-}$mice demonstrate less dilatation and better color preservation of the ligated kidneys compared with WT mice, indicating slower progression of fibrosis. C: Representative images of Sirius Red-stained kidney sections from WT and $C R T^{+/-}$mice at 8 and 17 days after UU0. Quantification of the staining showed substantially reduced collagen deposition in the kidneys of heterozygous mice at both time points. $n=3$ per group. The tubulointerstitial area stained was quantified in non-overlapping images of the entire cortex. ${ }^{* *} P<0.01$ versus WT; ${ }^{\dagger} P<0.05$ versus L8WT; ${ }^{\sharp \ddagger} P<0.01$ versus L17WT. Scale bars: $50 \mu \mathrm{m}$ (C). 
A

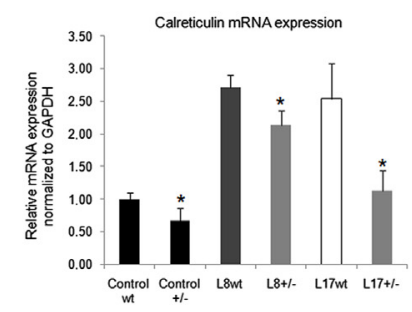

D

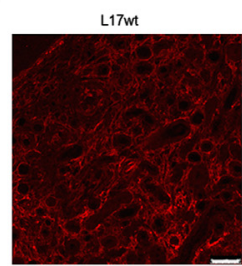

B mor

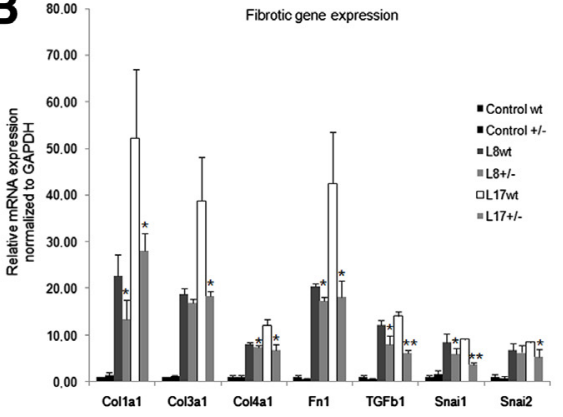

C
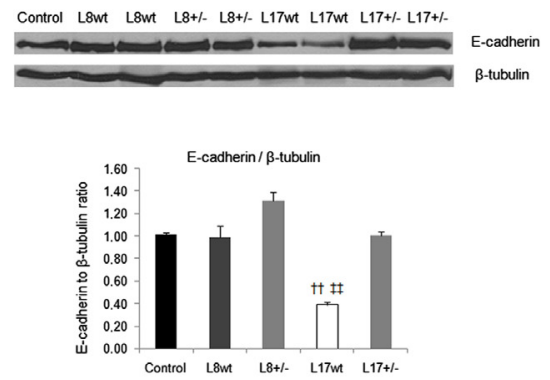

E
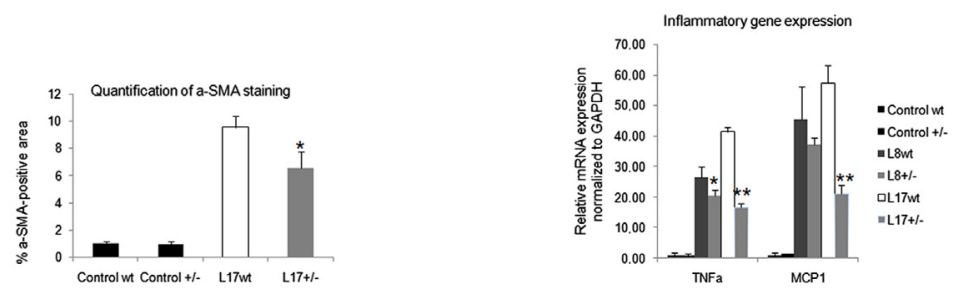

Figure 8 Decreased calreticulin (CRT) levels in heterozygous mice change the fibrotic profile in unilateral ureteric obstruction (UU0)-induced tubulointerstitial fibrosis. A: Relative mRNA amount (RT-qPCR) of the CRT gene in kidney homogenates of control kidneys at 8 and 17 days after UU0 in WT and $C R T^{+/-}$mice shows that CRT is significantly reduced in $C R T^{+/-}$compared with WT mice, with variable degree of reduction in control kidneys and kidneys at 8 and 17 days after UU0. B: Relative mRNA amount of key profibrotic genes (Col1a1, Col3a1, Col4a1, Fn1, TGFb1, Snai1, and Snai2) in WT and CRT ${ }^{+-}$mice at 8 or 17 days after UUO shows a small repression at 8 days and notably increased repression at 17 days in heterozygous mice, consistent with the expression pattern of CRT at the corresponding UUO time points. C: Representative blot of E-cadherin expression in WT and CRT $T^{+/-}$mice at 8 or 17 days after UU0 shows that E-cadherin is substantially reduced at 17 days in WT but not in $C R T^{+/-}$mice, implicating better preservation of the epithelial structure in heterozygous mice. D: Representative images of $\alpha$-SMA-stained kidney sections from WT and $C R T^{+/-}$mice at 17 days after UUO and quantification of the staining in non-overlapping images of the entire cortex shows significantly reduced $\alpha$-SMA-positive area in heterozygous mice. E: Relative mRNA amount of the proinflammatory genes TNF- $a$ and MCP1 in WT and $C R T^{1 /-}$ mice at 8 or 17 days after UUO shows that the proinflammatory mediators are repressed in heterozygous mice after UUO to a degree consistent with the level of CRT expression (E). $n=3$ per group. ${ }^{\star} P<0.05,{ }^{* *} P<0.01$ versus control WT, L8WT, or L17WT (A, B, D, and E); ${ }^{\dagger \dagger} P<0.01$ versus control (C); ${ }^{\ddagger \ddagger} P<0.01$ versus $\mathrm{L}^{\dagger} 7^{+/-}$(C). Scale bars: $50 \mu \mathrm{m}$ (D).

(Figure 4). ${ }^{18-20}$ That the low CRT overexpression caused a greater decrease in E-cadherin expression is in total agreement with the greater increase in Snail levels and stronger activation of the $\beta$-catenin pathway in the overexpression 1 clone (data not shown). These results may indicate that slight changes in CRT expression may be able to disproportionally affect certain pathways at the cell

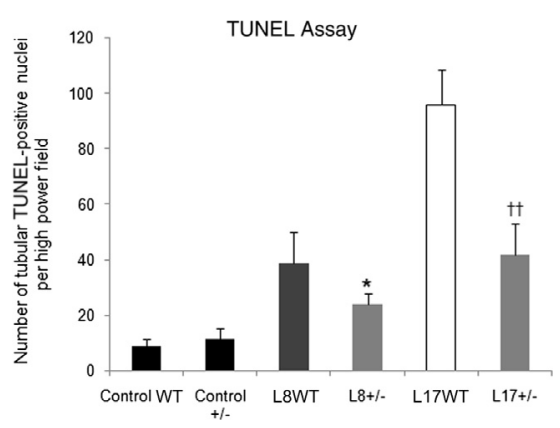

Figure 9 Decreased calreticulin (CRT) levels in heterozygous mice protect from tubular cell loss in unilateral ureteric obstruction (UUO)-induced tubular interstitial fibrosis. Number of tubular TUNEL-positive nuclei per high-power field in WT and $C R T^{+/-}$mice at 8 or 17 days after UUO. Tubular apoptosis is notably decreased in heterozygous mice at both time points. $n=3$ per group. Staining was quantified in 10 images at $\times 200$ magnification per animal. ${ }^{*} P<$ 0.05 versus $L 8 W T ;{ }^{\dagger \dagger} P<0.01$ versus L17WT. culture level and that fine tuning of CRT levels intracellularly may be important for cellular functions. However, in the case of a complex tissue in which other factors and cell types are also involved, these effects may be counterbalanced. In agreement with these findings, a previous study of Madin-Darby canine kidney cells evaluated the role of CRT in induction of EMT. ${ }^{26}$ EMT previously has been proposed to have a pivotal contribution to development of renal fibrosis. ${ }^{20,27}$ However, recent studies have challenged this notion and have rendered the role of EMT highly controversial. ${ }^{28,29}$ Interestingly, our results showed that CRT overexpression in TECs did not induce expression of either Acta2 (the gene coding for $\alpha$-SMA), the most commonly used marker for activated fibroblasts and myofibroblasts, or Collal, the major collagen type produced by fibroblasts. Previous studies of fibroblast cell lines have proportionally correlated CRT abundance with collagen I expression and deposition into the ECM. ${ }^{15}$ These results, combined with our findings, indicate that CRT may regulate certain cellular functions in a cell type-specific manner. For example, CRT up-regulation in TECs enhanced production of other ECM components including fibronectin and collagen IV, which are not fibroblast specific. These data suggest that CRT up-regulation initiates epithelial plasticity, which may facilitate tubular cells to adjust to their fibrotic 
A

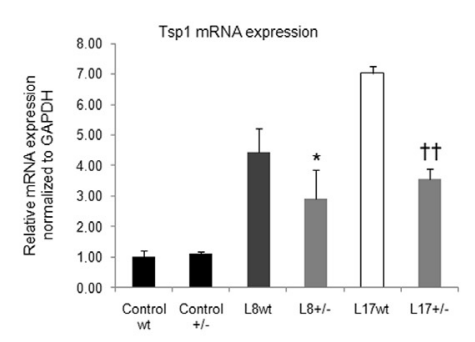

C

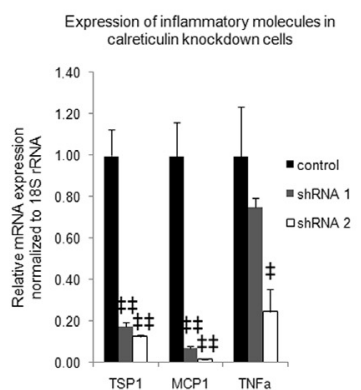

D

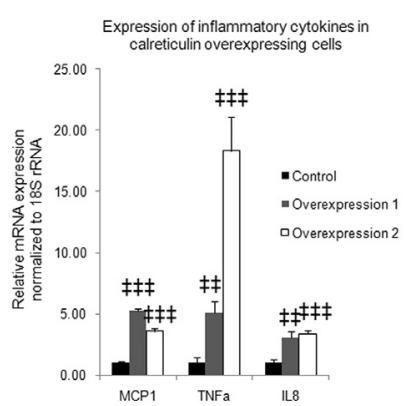

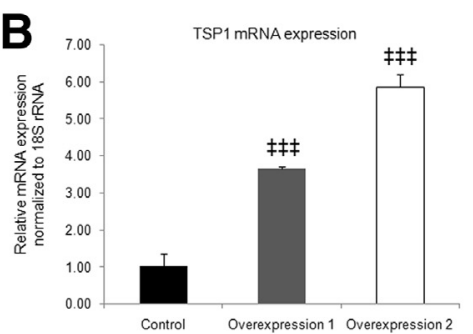

E

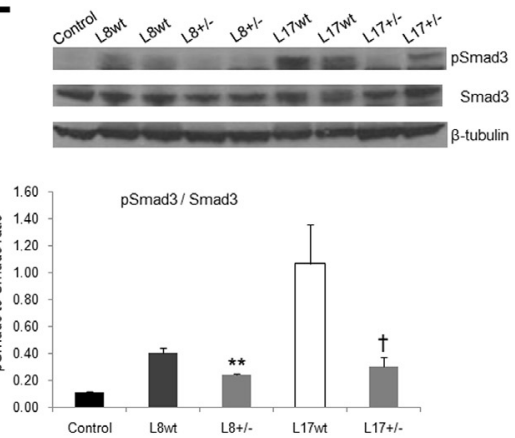

Figure 10 Calreticulin (CRT) levels affect molecules being upstream or downstream transforming growth factor $\beta 1$ (TGF- $\beta 1$ ) signaling. A: Relative mRNA amount of TSP1 in kidney homogenates of WT and $C R T^{+/-}$mice after 8 or 17 days of UUO shows that the TSP1 levels increase with progression of fibrosis but are significantly repressed in mice lacking one CRT allele at both time points examined. $n=3$ per group. B: Relative mRNA amount of TSP1 in tubular epithelial cells (TECs) overexpressing CRT shows up-regulation of TSP1. C: Relative mRNA amount of inflammatory molecules in CRT knockdown TECs shows significant suppression of TSP1, MCP1, and TNF- $a$. D: Relative mRNA increase in inflammatory cytokines MCP1, TNF- $\alpha$, and IL-8 in TECs overexpressing CRT links CRT upregulation with mediation of a proinflammatory state triggered by renal TECs. E: Relative mRNA amounts were determined via RT-qPCR. Representative blot shows reduction in pSmad3 expression in WT and $C R T^{+/-}$mice at 8 or 17 days after UU0, indicating an important attenuation of TGF- $\beta 1$ signaling. Data represent means \pm SD of three independent experiments. ${ }^{*} P<0.05,{ }^{*}{ }^{*} P<0.01$ versus L8WT $(\mathbf{A}$ and $\mathbf{E}) ;{ }^{\dagger} P<0.05,{ }^{\dagger \dagger} P<0.01$ versus L17WT $(\mathbf{A}$ and $\mathbf{E}) ;{ }^{\ddagger} P<$ $0.05,{ }^{\ddagger} P<0.01,{ }^{\ddagger \ddagger} P<0.001$ versus control (B-D).

environment; however, it is in itself insufficient for full myofibroblastic differentiation of epithelial cells, which can be promoted in vitro by other factors such as TGF- $\beta 1$. That CRT repression in TECs suppressed both TGF- $\beta 1$ responses and Collal expression shows that CRT promotes fibrotic pathways and that its reduction is able to attenuate expression of several fibrotic markers. In agreement with these findings, it was shown that knockdown of CRT in fibroblast cell lines attenuates TGF- $\beta 1$-induced ECM production. ${ }^{30}$

In accordance with this alteration of cell phenotype was acquisition of a more elongated architecture and increased motility of the CRT-overexpressing TECs. Previous studies of several cell lines have yielded contradictory results insofar as the role of CRT in wound healing and modulation of cell adhesiveness. ${ }^{31-35}$ For example, CRT overexpression in fibroblasts enhanced cell attachment efficacy on fibronectin-coated matrices and reduced cell motility. ${ }^{3}$ In contrast, in vitro treatment with exogenous CRT increased proliferation and migration of both epithelial cells and fibroblasts. ${ }^{35}$ The decreased proliferation rate of CRToverexpressing TECs in the present study can be attributed to the fact that endogenous ER resident CRT may exert different functions from exogenously added CRT. Furthermore, it is possible that the influence of CRT on cell motility differs between epithelial cells and fibroblasts. Induction of Tspl in CRT-overexpressing TECs, which promotes focal adhesion disassembly and cell migration, ${ }^{23,33}$ may have a role in the increased motility observed in CRT overexpressors.

ER stress and apoptosis are increasingly appreciated as major contributors to progression of renal fibrosis. ${ }^{21,22} \mathrm{CRT}$, apart from its other cellular functions, serves as an ER stress response protein, being up-regulated by various stress stimuli. $^{36,37}$ Moreover, separate studies of a variety of cell lines have attributed opposing roles to CRT in regulation of apoptosis. ${ }^{38-44}$ For example, certain studies have proportionally correlated CRT abundance with increased cell sensitivity to apoptosis, ${ }^{38-41}$ whereas others have positively associated CRT expression with protection from apoptosis induced by diverse stimuli such as nitric oxide, hypoxia, or intracellular $\mathrm{Ca}^{2+}$ overload. ${ }^{42-44}$ It is possible that the effect of CRT on viability and apoptosis differs according to the cell line used, the level of CRT expression, or the severity and duration of the apoptotic stimulus. In the present study, CRT overexpression in TECs aggravated the ER stress state and rendered the cells prone to apoptotic death. In addition to the data presented for GRP78, our preliminary studies indicated that phosphorylation of the eukaryotic initiation factor 2a (peIF2a), a crucial step in the stress-induced signaling pathways, ${ }^{16,17}$ is increased in CRT-overexpressing TECs (data not shown). CRT knockdown in TECs also induced prominent up-regulation of GRP78 expression. 
This is consistent with the increased expression of ER stress proteins, including GRP78 observed in CRT-null mouse embryonic fibroblasts. ${ }^{45}$ These data suggest that CRT deficiency triggers an ER stress response to compensate for accumulation of unfolded proteins and to facilitate the protein quality control chaperoned in the ER. In contrast, ER stress proteins also regulate fibrotic processes. ${ }^{46}$ Our results suggest that CRT overexpression in TECs induced an ER stress response, in particular accompanied by fibrotic changes and apoptosis, alterations that were not observed in CRT knockdown TECs despite increased expression of GRP78. Consistently, we confirmed the existence of broad ER stress in the CRT-overexpressing tubular cells in vivo in the UUO model, in which a high degree of co-distribution was noted. These observations support the idea that CRT may contribute to renal fibrosis through ER stress and apoptosis induction.

Because CRT up-regulation in TECs causes phenotypic alterations that may be considered crucial steps during the progression of fibrosis, can reduction of its expression have protective effects on fibrosis development? In that deletion of the $C R T$ gene causes embryonic lethality, ${ }^{14}$ the use of heterozygous transgenic mice, with an approximately $50 \%$ reduction in CRT expression, was an ideal tool for directly investigating the role of CRT in fibrosis. Although CRT was up-regulated in the UUO model, as expected, its expression in heterozygous mice was not proportionally reduced, showing a greater decrease at 17 days of obstruction. It is possible that the need for increased CRT expression led to greater up-regulation of the single CRT allele in heterozygous mice in the early stages of fibrosis, which could not be sustained to the later stages of the disease. Apparently, the reduced expression of CRT in heterozygous mice is not restricted to tubules, in which CRT is specifically upregulated in the UUO model of fibrosis. ${ }^{9}$ The possibility that decreased CRT expression in other cell types, including interstitial fibroblasts and immune cells, also affects the progression of fibrosis cannot be excluded. However, the specific function of CRT in these cell types has been extensively examined in other studies and is under discussion.

In accordance with our hypothesis, CRT deficiency notably improved the gross appearance of the ligated kidneys, seen macroscopically with less dilatation and better color preservation as compared with WT kidneys. This delay of the fibrotic process was further established and quantitated via Sirius Red staining, which verified that the kidneys of heterozygous mice had substantially reduced ECM accumulation at both time intervals (ie, starting at day 8 ) and that this reduction was even more dramatic at 17 days after UUO. The observation of a $35 \%$ reduction in Sirius Red staining at 8 days after UUO accompanied by a smaller $20 \%$ decrease in CRT transcript can be attributed to the fact that CRT expression level corresponds to the specific time point examined, whereas the area of Sirius Red staining represents the total amount of ECM accumulated from onset of the disease. Accordingly, the area of $\alpha$-SMA-positive myofibroblasts and the expression levels of all ECM molecules examined were attenuated in heterozygous mice, most significantly at 17 days after UUO, in accordance with the greater reduction in CRT levels. In addition to the decrease in ECM accumulation, CRT heterozygous mice exhibited better preservation of the tubular structure, evidenced by the maintenance of E-cadherin expression and attenuation of tubular apoptosis. Consistently, the transcriptional regulators of EMT and fibrosis, Snail and Snai2, were repressed in heterozygous mice. These results are confirmed by our findings in cultured TECs and suggest that CRT affects profibrotic pathways, at least in part, by inducing major transcriptional regulators of fibrosis such as Snail and Snai2.

A central mediator in these pathways is the profibrotic cytokine TGF- $\beta 1$, the expression of which was attenuated in CRT heterozygous mice. TGF- $\beta 1$ mediates several key pathologic events during CKD progression, including fibroblast activation, tubular and fibroblast ECM production, epithelial cell death, and inflammatory processes, and the disruption of its signaling impeded development of chronic renal damage. ${ }^{47,48}$ The major source of TGF- $\beta 1$ during fibrosis is the infiltrating inflammatory cells. ${ }^{25}$ Apparently, inflammation was attenuated in heterozygous mice, as demonstrated by the decrease in inflammatory mediators (MCPI and $T N F-\alpha)$ and the smaller perivascular infiltrates observed (data not shown). In TECs overexpressing CRT, several inflammatory cytokines were induced (MCP1,TNF- $\alpha$, and $I L-8)$, which suggests that epithelial up-regulation of CRT during fibrosis may be involved in establishment of the inflammatory state that characterizes the fibrotic kidney, thereby increasing TGF- $\beta 1$ production. Consistently, a number of immunologic functions have already been ascribed to CRT. Cell surface expression of CRT promotes the phagocytic uptake of dying cells, assists in maturation of immune cells, and enhances lymphocyte infiltration of tumors. ${ }^{49-52}$ That the cell surface form of CRT promotes clearance of apoptotic cells does not contradict the reduced tubular apoptosis observed in heterozygous mice because it is possible that the decrease in ER resident CRT attenuates tubular apoptosis. In addition, CRT possibly may regulate TGF- $\beta 1$ activation by directly affecting expression of TSP1, an endogenous TGF- $\beta 1$ activator, as shown by the prominent up-regulation of TSPI in TECs overexpressing CRT and the impressive suppression of TSPI in knockdown TECs. This result may seem contradictory to the increased levels of TSPI transcript observed in CRT-null mouse embryonic fibroblasts ${ }^{15}$; however, this may be another example of cell type-specific phenomenon, highlighting the difference between the responses of epithelial cells and fibroblasts. Moreover, the cell surface form of CRT binds to TSP1 and stimulates events associated with wound healing including cell migration, anoikis resistance, and matrix deposition, which may also represent a mechanism through which CRT affects 
fibrosis. $^{23,33,52}$ TSP1 expression was repressed in CRT heterozygous mice both at earlier and later intervals, implying that TGF- $\beta 1$ activation was alleviated. Reduced TGF- $\beta 1$ activation was evidenced by the significant decrease in phosphorylated Smad3 levels at both time points in heterozygous mice. Therefore, we suggest that CRT deficiency attenuates progression of fibrosis by suppressing both TGF- $\beta 1$ expression and activation and by hampering TGF- $\beta 1$ responses, as demonstrated in CRT knockdown TECs.

\section{Conclusion}

Results of the present study demonstrate that overexpression of CRT in TECs is a critical regulator of several aspects of tubulointerstitial fibrosis development. Reduction of CRT expression in transgenic heterozygous mice efficiently ameliorates progression of fibrosis by affecting inflammatory processes, TGF- $\beta 1$ production and/or activation, transcriptional regulation of fibrotic molecules, and apoptosis. The ability of CRT to affect so many aspects of fibrosis indicates that decreasing CRT levels may represent a novel targeted and effective treatment against fibrosis and CKD. The mechanisms of fibrosis development in various organs may have pathways shared in common or differing in a tissuespecific manner. For example, in an animal model of bleomycin-induced lung fibrosis, CRT was also up-regulated during fibrosis progression. ${ }^{9}$ It is therefore possible that in organs other than the kidney the fibrotic process could be also targeted by reduction of the levels of CRT.

\section{Acknowledgments}

We thank Dr. Demetrios Vlahakos for overseeing the project, Dr. Michalis Katsiboulas for valuable assistance with animal models, Dr. Stamatis Pagakis and Eleni Rigana for assistance with imaging, and Petros Moustardas for help with histologic techniques.

\section{Supplemental Data}

Supplemental material for this article can be found at http://dx.doi.org/10.1016/j.ajpath.2013.07.014.

\section{References}

1. de Jong PE, van der Velde M, Gansevoort RT, Zoccali C: Screening for chronic kidney disease: where does Europe go? Clin J Am Soc Nephrol 2008, 3:616-623

2. Coresh J, Selvin E, Stevens LA, Manzi J, Kusek JW, Eggers P, Van Lente F, Levey AS: Prevalence of chronic kidney disease in the United States. JAMA 2007, 298:2038-2047

3. Nath KA: Tubulointerstitial changes as a major determinant in the progression of renal damage. Am J Kidney Dis 1992, 20:1-17

4. Wynn TA: Common and unique mechanisms regulate fibrosis in various fibroproliferative diseases. J Clin Invest 2007, 117:524-529
5. Boor P, Ostendorf T, Floege J: Renal fibrosis: novel insights into mechanisms and therapeutic targets. Nat Rev Nephrol 2010, 6:643-656

6. Sivakumar P, Das AM: Fibrosis, chronic inflammation and new pathways for drug discovery. Inflamm Res 2008, 57:410-418

7. Bascands JL, Schanstra JP: Obstructive nephropathy: insights from genetically engineered animals. Kidney Int 2005, 68:925-937

8. Chevalier RL, Forbes MS, Thornhill BA: Ureteral obstruction as a model of renal interstitial fibrosis and obstructive nephropathy. Kidney Int 2009, 75:1145-1152

9. Kypreou KP, Kavvadas P, Karamessinis P, Peroulis M, Alberti A, Sideras P, Psarras S, Capetanaki Y, Politis PK, Charonis AS: Altered expression of calreticulin during the development of fibrosis. Proteomics 2008, 8:2407-2419

10. Johnson S, Michalak M, Opas M, Eggleton P: The ins and outs of calreticulin: from the ER lumen to the extracellular space. Trends Cell Biol 2001, 11:122-129

11. Qiu Y, Michalak M: Transcriptional control of the calreticulin gene in health and disease. Int J Biochem Cell Biol 2009, 41:531-538

12. Lynch JM, Chilibeck K, Qui Y, Michalak M: Assembling pieces of the cardiac puzzle: calreticulin and calcium-dependent pathways in cardiac development, health, and disease. Trends Cardiovasc Med 2006, 16:65-69

13. Nakorchevsky A, Hewel JA, Kurian SM, Mondala TS, Campbell D, Head SR, Marsh CL, Yates JR 3rd, Salomon DR: Molecular mechanisms of chronic kidney transplant rejection via large-scale proteogenomic analysis of tissue biopsies. J Am Soc Nephrol 2010, 21: $362-373$

14. Mesaeli N, Nakamura K, Zvaritch E, Dickie P, Dziak E, Krause KH, Opas M, MacLennan DH, Michalak M: Calreticulin is essential for cardiac development. J Cell Biol 1999, 144:857-868

15. Van Duyn Graham L, Sweetwyne MT, Pallero MA, MurphyUllrich JE: Intracellular CRT regulates multiple steps in fibrillar collagen expression, trafficking, and processing into the extracellular matrix. J Biol Chem 2010, 285:7067-7078

16. Bánhegyi G, Baumeister P, Benedetti A, Dong D, Fu Y, Lee AS, Li J, Mao C, Margittai E, Ni M, Paschen W, Piccirella S, Senesi S, Sitia R, Wang M, Yang W: Endoplasmic reticulum stress. Ann NY Acad Sci 2007, 1113:58-71

17. Xu C, Bailly-Maitre B, Reed JC: Endoplasmic reticulum stress: cell life and death decisions. J Clin Invest 2005, 115:2656-2664

18. Boutet A, De Frutos CA, Maxwell PH, Mayol MJ, Romero J, Nieto MA: Snail activation disrupts tissue homeostasis and induces fibrosis in the adult kidney. EMBO J 2006, 25:5603-5613

19. Medici D, Hay ED, Olsen BR: Snail and Slug promote epithelialmesenchymal transition through beta-catenin-T-cell factor-4dependent expression of transforming growth factor-beta3. Mol Biol Cell 2008, 19:4875-4887

20. Kalluri R, Neilson EG: Epithelial-mesenchymal transition and its implications for fibrosis. J Clin Invest 2003, 112:1776-1784

21. Inagi R: Endoplasmic reticulum stress in the kidney as a novel mediator of kidney injury. Nephron Exp Nephrol 2009, 112:e1-e9

22. Docherty NG, O’Sullivan OE, Healy DA, Fitzpatrick JM, Watson RW: Evidence that inhibition of tubular cell apoptosis protects against renal damage and development of fibrosis following ureteric obstruction. Am J Physiol Renal Physiol 2006, 290:F4-F13

23. Sweetwyne MT, Murphy-Ullrich JE: Thrombospondin1 in tissue repair and fibrosis: TGF- $\beta$-dependent and independent mechanisms. Matrix Biol 2012, 31:178-186

24. Bige N, Shweke N, Benhassine S, Jouanneau C, Vandermeersch S, Dussaule JC, Chatziantoniou C, Ronco P, Boffa JJ: Thrombospondin-1 plays a profibrotic and pro-inflammatory role during ureteric obstruction. Kidney Int 2012, 81:1226-1238

25. Grande MT, Pérez-Barriocanal F, López-Novoa JM: Role of inflammation in tubulo-interstitial damage associated to obstructive nephropathy. J Inflamm (Lond) 2010, 7:19

26. Hayashida Y, Urata Y, Muroi E, Kono T, Miyata Y, Nomata K, Kanetake H, Kondo T, Ihara Y: Calreticulin represses E-cadherin 
gene expression in Madin-Darby canine kidney cells via Slug. J Biol Chem 2006, 281:32469-32484

27. Iwano M, Plieth D, Danoff TM, Xue C, Okada H, Neilson EG: Evidence that fibroblasts derive from epithelium during tissue fibrosis. J Clin Invest 2002, 110:341-350

28. Lin SL, Kisseleva T, Brenner DA, Duffield JS: Pericytes and perivascular fibroblasts are the primary source of collagen-producing cells in obstructive fibrosis of the kidney. Am J Pathol 2008, 173:1617-1627

29. Grgic I, Duffield JS, Humphreys BD: The origin of interstitial myofibroblasts in chronic kidney disease. Pediatr Nephrol 2012, 27 : 183-193

30. Zimmerman KA, Graham LV, Pallero MA, Murphy-Ullrich JE: Calreticulin regulates transforming growth factor- $\beta$-stimulated extracellular matrix production. J Biol Chem 2013, 288:14584-14598

31. Opas M, Szewczenko-Pawlikowski M, Jass GK, Mesaeli N, Michalak M: Calreticulin modulates cell adhesiveness via regulation of vinculin expression. J Cell Biol 1996, 135:1913-1923

32. Coppolino MG, Woodside MJ, Demaurex N, Grinstein S, StArnaud R, Dedhar S: Calreticulin is essential for integrin-mediated calcium signalling and cell adhesion. Nature 1997, 386:843-847

33. Goicoechea S, Orr AW, Pallero MA, Eggleton P, Murphy-Ullrich JE: Thrombospondin mediates focal adhesion disassembly through interactions with cell surface calreticulin. J Biol Chem 2000, 275: 36358-36368

34. Papp S, Szabo E, Kim H, McCulloch CA, Opas M: Kinase-dependent adhesion to fibronectin: regulation by calreticulin. Exp Cell Res 2008, 314:1313-1326

35. Nanney LB, Woodrell CD, Greives MR, Cardwell NL, Pollins AC, Bancroft TA, Chesser A, Michalak M, Rahman M, Siebert JW, Gold LI: Calreticulin enhances porcine wound repair by diverse biological effects. Am J Pathol 2008, 173:610-630

36. Waser M, Mesaeli N, Spencer C, Michalak M: Regulation of calreticulin gene expression by calcium. J Cell Biol 1997, 138:547-557

37. Yamazaki T, Muramoto M, Nishimura S, Kita Y: Suppressive effects of FR167653, an inhibitor of p38 mitogen-activated kinase, on calreticulin mRNA expression induced by endoplasmic reticulum stresses. Eur J Pharmacol 2004, 484:147-156

38. Nakamura K, Bossy-Wetzel E, Burns K, Fadel MP, Lozyk M, Goping IS, Opas M, Bleackley RC, Green DR, Michalak M: Changes in endoplasmic reticulum luminal environment affect cell sensitivity to apoptosis. J Cell Biol 2000, 150:731-740

39. Mesaeli N, Phillipson C: Impaired p53 expression, function, and nuclear localization in calreticulin-deficient cells. Mol Biol Cell 2004, 15:1862-1870
40. Ihara Y, Urata Y, Goto S, Kondo T: Role of calreticulin in the sensitivity of myocardiac $\mathrm{H} 9 \mathrm{c} 2$ cells to oxidative stress caused by hydrogen peroxide. Am J Physiol Cell Physiol 2006, 290:C208-C221

41. Waterhouse NJ, Pinkoski MJ: Calreticulin: raising awareness of apoptosis. Apoptosis 2007, 12:631-634

42. Jia L, Xu M, Zhen W, Shen X, Zhu Y, Wang W, Wang X: Novel antioxidative role of calreticulin in protecting A549 human type II alveolar epithelial cells against hypoxic injury. Am J Physiol Cell Physiol 2008, 294:C47-C55

43. Oyadomari S, Takeda K, Takiguchi M, Gotoh T, Matsumoto M, Wada I, Akira S, Araki E, Mori M: Nitric oxide-induced apoptosis in pancreatic beta cells is mediated by the endoplasmic reticulum stress pathway. Proc Natl Acad Sci U S A 2001, 98:10845-10850

44. Zhu N, Wang Z: Calreticulin expression is associated with androgen regulation of the sensitivity to calcium ionophore-induced apoptosis in LNCaP prostate cancer cells. Cancer Res 1999, 59:1896-1902

45. Knee R, Ahsan I, Mesaeli N, Kaufman RJ, Michalak M: Compromised calnexin function in calreticulin-deficient cells. Biochem Biophys Res Commun 2003, 304:661-666

46. Baek HA, Kim do S, Park HS, Jang KY, Kang MJ, Lee DG, Moon WS, Chae HJ, Chung MJ: Involvement of endoplasmic reticulum stress in myofibroblastic differentiation of lung fibroblasts. Am J Respir Cell Mol Biol 2012, 46:731-739

47. López-Hernández FJ, López-Novoa JM: Role of TGF- $\beta$ in chronic kidney disease: an integration of tubular, glomerular and vascular effects. Cell Tissue Res 2012, 347:141-154

48. Sato M, Muragaki Y, Saika S, Roberts AB, Ooshima A: Targeted disruption of TGF-beta1/Smad3 signaling protects against renal tubulointerstitial fibrosis induced by unilateral ureteral obstruction. J Clin Invest 2003, 112:1486-1494

49. Raghavan M, Wijeyesakere SJ, Peters LR, Del Cid N: Calreticulin in the immune system: ins and outs. Trends Immunol 2013, 34:13-21

50. Pawaria S, Binder RJ: CD91-dependent programming of T-helper cell responses following heat shock protein immunization. Nat Commun $2011,2: 521$

51. Wang HT, Lee HI, Guo JH, Chen SH, Liao ZK, Huang KW, Torng PL, Hwang LH: Calreticulin promotes tumor lymphocyte infiltration and enhances the antitumor effects of immunotherapy by up-regulating the endothelial expression of adhesion molecules. Int $\mathrm{J}$ Cancer 2012, 130:2892-2902

52. Gold LI, Eggleton P, Sweetwyne MT, Van Duyn LB, Greives MR, Naylor SM, Michalak M, Murphy-Ullrich JE: Calreticulin: nonendoplasmic reticulum functions in physiology and disease. FASEB J 2010, 24:665-683 